\title{
LUNG NEOPLASM WITH HYPERTROPHIC OSTEOARTHROPATHY SIMULATING RHEUMATOID ARTHRITIS: CASE REPORT
}

\begin{abstract}
Robson Antônio Gonçalves ${ }^{1}$, Gabriela Almeida Barbosa ${ }^{1}$, Tâmara Santos Melo ${ }^{1}$, Valéria Bezerra da Silva ${ }^{1}$, Sandra Rejane Cabral Batista ${ }^{1}$, Danielle Christinne Soares Egypto ${ }^{1}$, Ana Karla Guedes de Melo ${ }^{1}$, Maria Roberta Melo Pereira Soares ${ }^{1, \star}$, Alessandra de Sousa Braz ${ }^{1}$, Eutilia Andrade Medeiros Freire ${ }^{1}$
\end{abstract}

1.Universidade Federal da Paraíba, João Pessoa (PB), Brazil.

*Corresponding author: mariarobertam@hotmail.com

\section{BACKGROUND}

Hypertrophic osteoarthropathy (HOA) is a syndrome and the secondary form is the most prevalent, present for up to $97 \%$ of cases. It is characterized by arthritis, digital clubbing, abnormal skin proliferation and periostitis of tubular bones. Benign pathologies such as heart disease, sarcoidosis, inflammatory bowel disease, liver cirrhosis and lung infections are related to the disease; however, lung cancer is the most prevalent form, present in 0.2 to $17 \%$ of patients. Among lung neoplasms, the non-small cell histological type is the most associated, considered a paraneoplastic rheumatic syndrome. Treatment is related to the underlying disease. Hypertrophic osteoarthropathy should be included in the differential diagnosis of sudden-onset exuberant synovitis in individuals over 50 years of age. Rheumatoid factor (RF), antinuclear antibodies (ANA) and antibodies against citrullinated peptides may be present, being a differential diagnosis of rheumatoid arthritis (RA).

\section{CASE REPORT}

Female, 64 years old, smoker, with diabetes and osteoporosis, history of additive and symmetrical polyarthritis of the hands, wrists, elbows, knees and ankles of a progressive character with a 1-year evolution, associated with joint stiffness, asthenia, nail changes and skin thickening in hands. Recent diagnosis of seronegative RA, using methotrexate $15 \mathrm{mg} / \mathrm{day}$ for 2 weeks. Hospital admission (December 2020) due to mucositis and persistence of arthritis, which worsened in the last 4 weeks. On physical examination, arthritis in hands, wrists, elbows, knees, ankles and feet, associated with clubbing (hands/feet) and palmar hyperkeratosis (Figures 1 and 2). Exams with bicytopenia (anemia and leukopenia) and thrombocytosis. Renal, liver and thyroid function preserved, C-reactive protein $81.8 \mathrm{mg} / \mathrm{L}$. ANA 1:320 (nuclear dotted centromeric), RF negative. Negative serologies (HIV/hepatitis). Radiographs showing periosteal reactions in the proximal phalanges of the hands, humeral shafts, ulnas, femurs, tibias and fibulas (Figures 3-5). Chest tomography: solid mass with irregular and spiculated margins in the left lower lobe measuring $6.5 \times 5.4 \times 5.3 \mathrm{~cm}$. Diagnostic hypothesis of lung cancer with hypertrophic osteoarthropathy simulating RA. Initial treatment with prednisone $10 \mathrm{mg} /$ day, analgesics, risedronate $35 \mathrm{mg} /$ week, supplementation (calcium/vitamin D) and methotrexate suspension. After 1 week, partial improvement of pain complaints with complete resolution of mucositis. In the oncological and surgical evaluation continued with the investigation with lung biopsy, a result compatible with poorly differentiated non-small cell carcinoma.

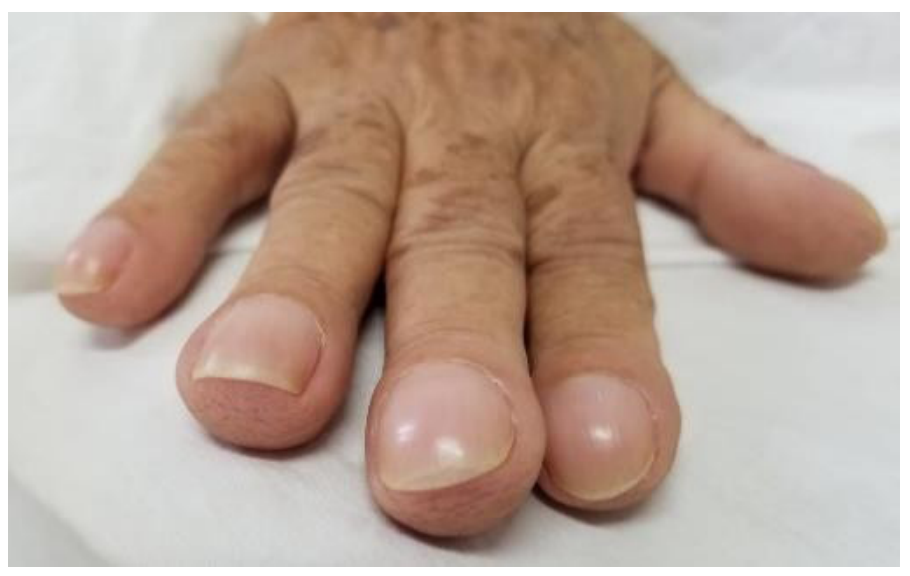

Figure 1.

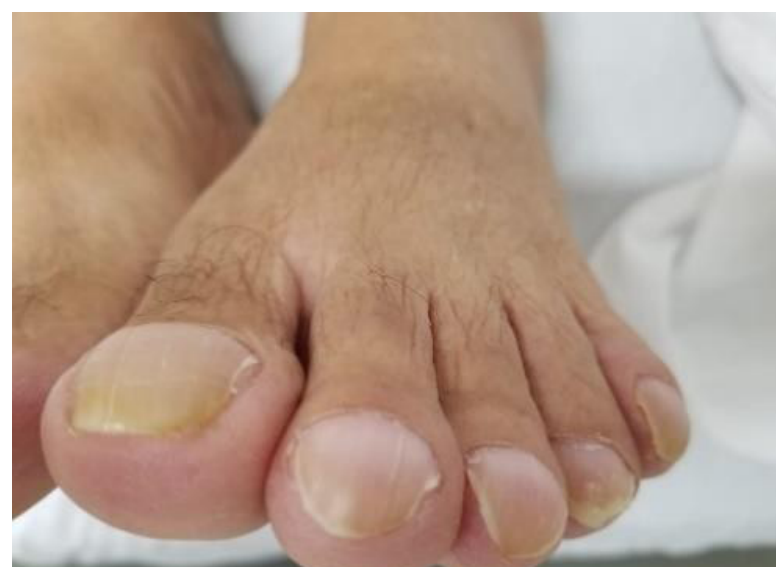

Figure 2.

Realização: 


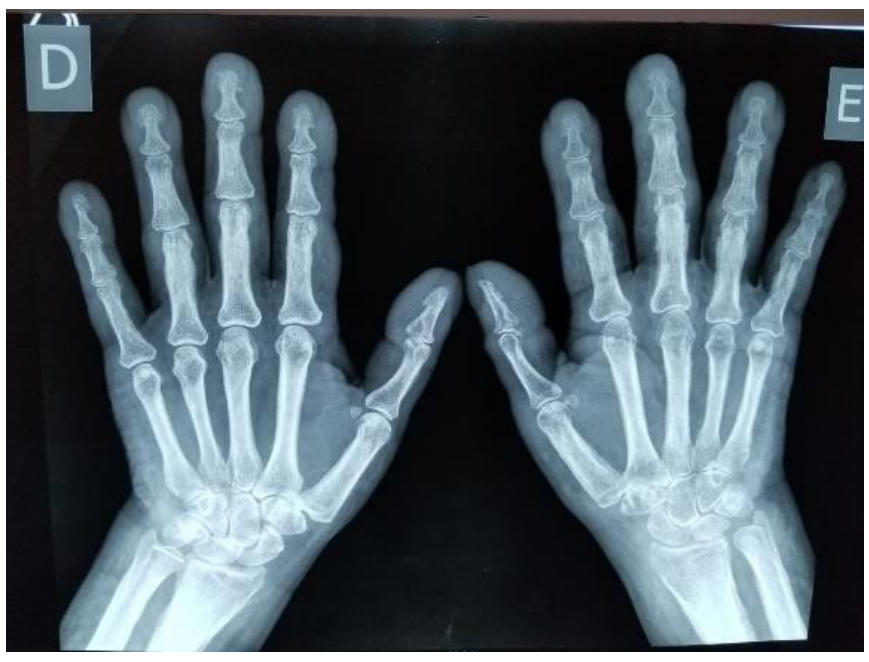

Figure 3

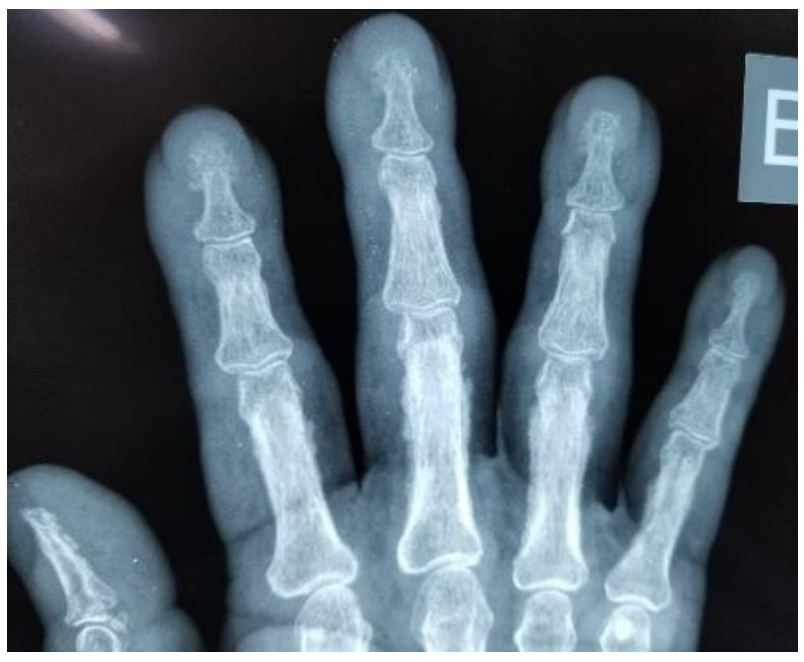

Figure 4.

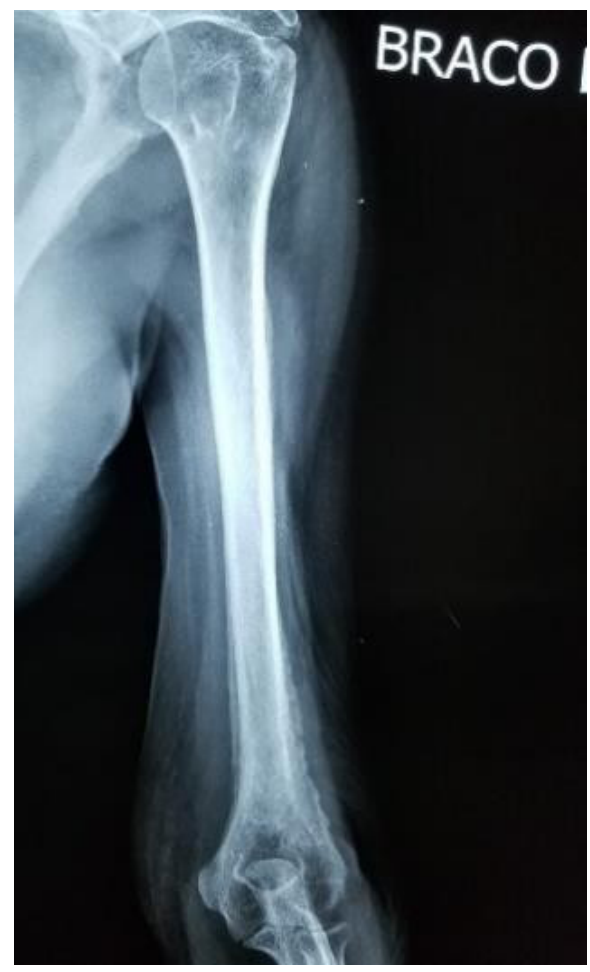

Figure 5.

\section{CONCLUSION}

The authors draw attention to the exuberant condition of arthritis in which the patient was already starting therapy for RA. A wide investigation is of paramount importance, as the patient can be spared from unnecessary rheumatologic therapies, delay in cancer diagnosis and appropriate therapy.

\section{KEYWORDS}

Hypertrophic osteoarthropathy, Lung cancer, Rheumatoid arthritis. 